\title{
A FAST FAULT DETECTION SCHEME FOR POWER CONVERTERS IN DISTRIBUTED GENERATION SYSTEMS
}

\author{
Gustavo M. S. Azevedo, Marcelo C. Cavalcanti, Francisco A. S. Neves, Fabricio Bradaschia \\ Federal University of Pernambuco (UFPE - DEE), Recife - Brazil \\ gustavomsa@aim.com,marcelo.cavalcanti@ufpe.br
}

\begin{abstract}
In this paper, a fault detection algorithm based on voltage disturbances is proposed and analyzed. This method observes the voltage characteristics instead of the current amplitude, that is the most common approach in power distribution electrical systems. The proposed system uses a dual quadrature signal generator for each voltage component in a stationary reference frame to extract its positive- and negative-sequence components. Furthermore, a frequency-locked loop is used to estimate the voltage frequency. The voltage amplitude and frequency instantaneous values are compared with the nominal counterparts by means of hysteresis comparators. As a result, a fast and reliable fault detector system is obtained. Simulations and experimental results are presented to illustrate the feasibility and performance of the proposed scheme.
\end{abstract}

\section{Keywords - Microgrids, Distributed Generation} Systems.

\section{INTRODUCTION}

Due to the depletion of oil resources and environmental concerns, there is an increasing tendency of generating electrical energy locally, at distribution level, by using renewable energy sources like natural gas, fuel cells, solar photovoltaic cells, wind power, and so on. According to the International Energy Outlook 2010 [1], the fastest growing sources of electricity generation are renewable. Among these sources, the ones which tend to experience biggest average annual percent increases until 2035 are photovoltaic and wind power, with projected annual rates of $12.7 \%$ and $7.8 \%$, respectively. The number of distributed generation (DG) and distributed storage (DS) units connected to distribution power grids through static converters is thus increasing fast. When these units can operate either connected to the grid or in islanded autonomous mode, supplying local loads during faults in the main grid, they form a microgrid. A microgrid must also be capable of riding-through between these two operating modes [2].

DG systems are generally modular small capacity plants, geographically widespread and located close to the loads. Thus, it is easy to find sites for them, reducing the construction time, cost and transmission losses [3]. However, there are difficulties in terms of power management of a microgrid, due to the the presence of small DG units with different rated power and generation characteristics, the absence of a strong energy generation source during islanded operation

Manuscript received on 16/03/2012. Revised on 06/06/2012. Accepted on $15 / 06 / 2012$ by recommendation of the Editor Henrique A. C. Braga. and also because of different control requirements of the power electronic interfaces for grid-connected or autonomous operation of the microgrid [2].

The control of a microgrid and its DG units is complex and much work on this subject is under progress. The main goal is installing new DG units without changing the whole control scheme of the microgrid or any pre-existing DG. As an approach to achieve this goal some authors have proposed to adapt the well known droop control scheme used in parallel synchronous generators for distributing the required load real and reactive power among the many DG units [4], [5]. Nevertheless, since the impedance of typical distribution feeders are not predominantly inductive, decoupled control of real and reactive power is not easily achieved, besides some stability problems that may occur [6], [7]. Further difficulties are expected due to significant differences in the impedances between the DG units and the microgrid or if there are local loads near to DG units.

Alternatives to solve this problem are based on a virtual active-reactive power reference frame [8] or a virtual frequency-voltage reference frame [9]. These transformations allow decoupled active and reactive power droop control. In order to improve the performance of active and reactive power sharing, application of the resistive droop method to parallel inverters has been investigated [10].

A major concern when defining the microgrid power management strategy is related to the transition from connected to autonomous operation mode and vice-versa. Most DG units are connected to the grid through power electronic converters. These converters are controlled so that their contribution to fault currents are not far above their rated value, differently from synchronous generators. This constraint makes difficult the use of conventional current-based fault detection schemes to change from utilityconnected to islanded operation mode. Moreover, according to SEMI fault ride-through capability curves [11], [12], many industrial loads would require a separation time of less than $50 \mathrm{~ms}$ after the occurrence of an abnormal condition in the grid. These times are not achievable with the current protection relays used in distribution systems [3], [13]. Another important issue is the detection that the grid normal operating condition has been restored and a scheme for synchronization of the microgrid with the utility voltage is necessary for the automatic reconnection. Due to the mentioned reasons, the main grid operating condition is generally determined by monitoring the grid voltages at the point of common coupling (PCC).

In this paper, a fast and reliable scheme for detecting the need for changing from grid-connected operation to islanded mode is proposed and analyzed. The method is based on a dual 
second order generalized integrator (DSOGI) and a frequencylocked loop (FLL) for determining the system frequency and separating the positive- and negative-sequence fundamentalfrequency voltage vector components at the PCC. These quantities are then used for recognizing grid faulty conditions and commanding the microgrid disconnection in about one fundamental period. Simulation and experimental results are presented to illustrate the feasibility and performance of the proposed schemes.

It is worth to point out that the fault detection presented here is one of the microgrid protection schemes and it must not be the single protection used. Current based relays are still necessary to cover fault conditions that do not cause sufficient disturbances in the voltages at the PCC to be detected by the proposed system.

\section{DESIGN OF THE FAULT DETECTION SYSTEM}

The proposed fault detection system is based on the characteristics of the voltage at the converter point of connection. The system extracts the positive- and negativesequence components of the voltage and check if they do not exceed permissible values. Furthermore, the frequency is also estimated and compared with its permissible values. The structures used to extract the voltage components and frequency as well as the limit comparators are described in the following.

\section{A. Positive- and Negative-Sequence Detection}

The concept of symmetrical components was proposed by Fortescue in 1918 [14] giving a new viewpoint for analysis of polyphase systems under unbalanced conditions. After that, Lyon extended this concept for the time-domain [15]. According to Lyon, the positive- and negative-sequence components of a three-phase voltage vector, $\vec{v}=\left[\begin{array}{lll}v_{a} & v_{b} & v_{c}\end{array}\right]^{T}$, are given by

$$
\begin{aligned}
& \vec{v}^{+}=\left[T^{+}\right] \vec{v}=\frac{1}{3}\left[\begin{array}{ccc}
1 & \alpha & \alpha^{2} \\
\alpha^{2} & 1 & \alpha \\
\alpha & \alpha^{2} & 1
\end{array}\right] \vec{v} \\
& \vec{v}^{-}=\left[T^{-}\right] \vec{v}=\frac{1}{3}\left[\begin{array}{ccc}
1 & \alpha^{2} & \alpha \\
\alpha & 1 & \alpha^{2} \\
\alpha^{2} & \alpha & 1
\end{array}\right] \vec{v}
\end{aligned}
$$

where $\alpha=e^{j 2 \pi / 3}$. In an ortogonal $\alpha \beta$ reference frame this equation results in

$$
\begin{aligned}
& \vec{v}_{\alpha \beta}^{+}=\frac{1}{2}\left[\begin{array}{cc}
1 & -q \\
q & 1
\end{array}\right] \vec{v}_{\alpha \beta} \\
& \vec{v}_{\alpha \beta}^{-}=\frac{1}{2}\left[\begin{array}{cc}
1 & q \\
-q & 1
\end{array}\right] \vec{v}_{\alpha \beta}
\end{aligned}
$$

where $q=e^{j \pi / 2}$ represents a $90^{\circ}$ phase-shift operator, which can be obtained through a quadrature signal generator based on a second order generalized integrator (SOGI) [16]. Therefore, two SOGI are enough to obtain the quadrature components necessary to perform (3) and (4) as shown in Figure 1 [17]. The transfer functions of the outputs of each
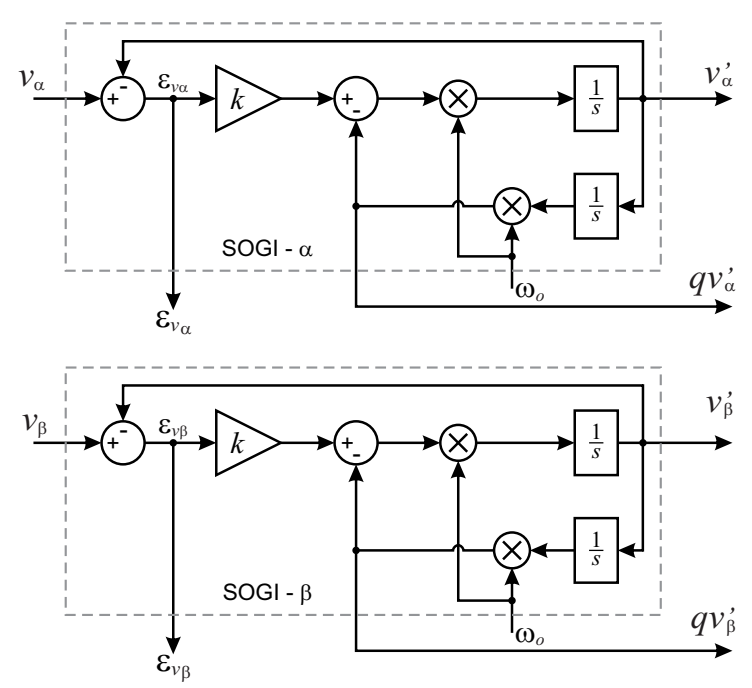

Fig. 1. Quadrature signal generation with SOGI.

SOGI are given by

$$
\begin{aligned}
& D(s)=\frac{v^{\prime}(s)}{v(s)}=\frac{k \omega_{o} s}{s^{2}+k \omega_{o} s+\omega_{o}^{2}} \\
& Q(s)=\frac{q v^{\prime}(s)}{v(s)}=\frac{k \omega_{o}^{2}}{s^{2}+k \omega_{o} s+\omega_{o}^{2}}
\end{aligned}
$$

where $\omega_{o}$ is the SOGI resonant frequency, which must match the grid frequency, and $k$ is the damping factor that defines the system dynamics.

The frequency response of $D(s)$ shows that it behaves as a band-pass filter with bandwidth dependent exclusively on $k$. On the other hand, $Q(s)$ behaves as a low-pass filter. The phase-angle between $v^{\prime}(t)$ and $q v^{\prime}(t)$ is always $90^{\circ}$ for any input frequency.

The SOGI of Figure 1 provide the signals necessary to calculate the positive- and negative-sequence components using (3) and (4), respectively. The magnitudes of these components are given by

$$
\begin{aligned}
& V^{+}=\left|\vec{v}_{\alpha \beta}^{+}\right|=\sqrt{\left(v_{\alpha}^{+}\right)^{2}+\left(v_{\beta}^{+}\right)^{2}}, \\
& V^{-}=\left|\vec{v}_{\alpha \beta}^{-}\right|=\sqrt{\left(v_{\alpha}^{-}\right)^{2}+\left(v_{\beta}^{-}\right)^{2}} .
\end{aligned}
$$

These variables and the estimated frequency are compared with their respective limits, and if any of them are out of their limits a fault condition is set.

An important issue in protection systems is its time response. Therefore, it is necessary to evaluate the SOGI dynamics in order to determine the behavior of the proposed system. Consider the structure of the SOGI from Figure 1 with $\omega_{o}$ constant, i.e., $\omega_{o}$ constant in (5) and (6). In addition, assume a sinusoidal input signal given by $v(t)=u(t) V \sin \omega t$, where $u(t)$ is the unit step function and $V$ is a constant. The 
time response of the SOGI outputs will be

$$
\begin{aligned}
v^{\prime}(t)= & V \sin (\omega t)-\frac{V}{\lambda} \sin (\lambda \omega t) e^{-\frac{k \omega_{0}}{2} t} \\
q v^{\prime}(t)= & V \cos (\omega t)- \\
& V\left[\cos (\lambda \omega t)+\frac{k}{2 \lambda} \sin (\lambda \omega t)\right] e^{-\frac{k \omega_{0}}{2} t}
\end{aligned}
$$

where $\lambda=\sqrt{4-k^{2}} / 2$ for $k<2$. Equations (9) and (10) reveal that, for $t \rightarrow \infty$, the SOGI outputs become $v^{\prime}(t)=v(t)$ and $q v^{\prime}(t)=v(t+\pi /(2 \omega))$, since the exponential parts become null. Besides, note that the time constant of the exponential part is $2 /\left(k \omega_{o}\right)$. Hence the settling time of both outputs is

$$
t_{s_{S O G I}}=\frac{8}{k \omega_{o}}
$$

The angular frequency $\omega_{o}$ does not deviate much from the nominal value, and then it can be considered a constant in (11). Thus, the settling time depends only on $k$. A high value of $k$ makes the SOGI faster, as shown in (11), however less immune to voltage harmonics and noise. Considering $k=\sqrt{3}$, $\omega_{o}=2 \pi f_{o}$ and $f_{o}=50 \mathrm{~Hz}$, it results $t_{s_{S O G I}} \approx 15 \mathrm{~ms}$ (less than one fundamental cycle). It is worth to point out that this analysis takes into account the influence of just one SOGI. Nevertheless, two SOGI and non-linear calculations, given by (7) and (8), are necessary to determine $V^{+}$and $V^{-}$. Even so, $t_{s_{S O G I}}$, given by (11), is still a good approximation of the settling time of signals $V^{+}$and $V^{-}$.

The positive- and negative-sequence components detected by the DSOGI are coupled during transients as shown in Figure 2. In this example, the voltage positive-sequence drops $0.1 \mathrm{pu}$ at $t=50 \mathrm{~ms}$, while the negative-sequence component is kept null. However, $V^{-}$is disturbed and its value reaches $0.035 \mathrm{pu}$ during this transient. At $t=150 \mathrm{~ms}$, the voltage negative-sequence rises to $0.1 \mathrm{pu}$, while the positivesequence component is kept constant. Nevertheless, $V^{+}$is also disturbed due to the coupling. Therefore, a low passfilter (LPF) is placed in each output $\left(V^{+}\right.$and $\left.V^{-}\right)$to reduce the effect of this coupling. $2^{\text {nd }}$-order Bessel filters are used due to its fast rise time with low overshoot and its outputs are designated as $V_{f}^{+}$and $V_{f}^{-}$. These filters are also able to mitigate the influence of voltage harmonics or noise that were not completely filtered by the SOGI. Besides, they can be used to adjust the protection response time, i.e., it is possible to increase the operation time by choosing a more selective filter. The filters used to obtain the results shown in Figure 2 have settling time of $15 \mathrm{~ms}$. Note that both $V_{f}^{+}$and $V_{f}^{-}$take around $20 \mathrm{~ms}$ to get stabilized.

It should be noted that the fault detection time also depends on the fault magnitude, i.e., severe faults, which result in high voltage drops or frequency deviation, are detected faster.

\section{B. Frequency Estimation}

The magnitudes of the voltage components will be correct when $\omega_{0}$ is equal to the grid frequency $\omega$. Therefore, a frequency adaptation system to tune the SOGI with the grid frequency is necessary. The FLL shown in Figure 3 is used for this purpose [17]. The signals $q v_{x}^{\prime}$ and $\varepsilon_{v_{x}}$ in Figure 1
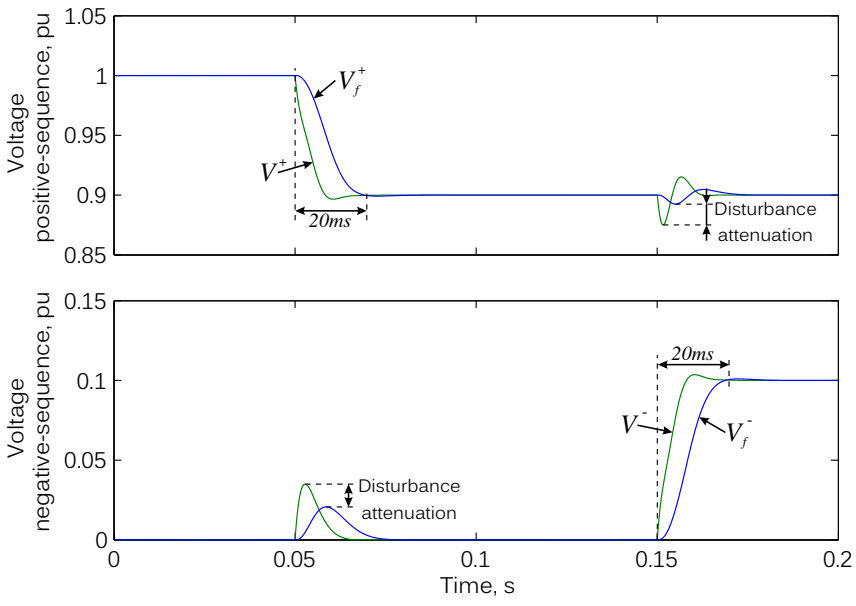

Fig. 2. Positive and negative-sequence components detected by the DSOGI and its attenuation with $2^{\text {nd }}$-order LPF.

are in phase when $\omega<\omega_{0}$ and they are in counter-phase when $\omega>\omega_{o}$. Therefore, a frequency error variable $\varepsilon_{f_{x}}$ is defined as the product of $q v_{x}^{\prime}$ by $\varepsilon_{v_{x}}$ of each SOGI ( $\alpha$ and $\beta)$. The average value of $\varepsilon_{f_{x}}$ is positive when $\omega<\omega_{o}$, zero when $\omega=\omega_{o}$ and negative when $\omega>\omega_{o}$. Hence, an integral controller with a negative gain $\Gamma$ is used to make the average component of $\varepsilon_{f}$ converge to zero by shifting the SOGI resonance frequency $\omega_{0}$ [18]. In the FLL shown in Figure $3, \Gamma$ is an integration gain and $\omega_{f f}$ is a feedforward term equal to the system nominal frequency. As it was shown in [18], the averaged dynamics of the FLL has a settling time that can be approximated by

$$
t_{s_{F L L}}=\frac{5}{\Gamma}
$$

The correct frequency estimation needs at least one fundamental cycle. Thus, the FLL dynamics should be slow. In this paper, it is chosen $\Gamma=125$, which results in $t_{s_{F L L}}=40 \mathrm{~ms}$ (two fundamental cycles). Furthermore, the estimated frequency, $\omega_{o}$, oscillates considerably during voltage magnitude or frequency transients. Therefore, a $2^{\text {nd }}$ order Bessel filter is used at the FLL output to obtain the filtered component, $\omega_{o_{f}}$. Due to the slow dynamics of the frequency estimation, the settling time of this filter is set to $80 \mathrm{~ms}$ (twice $t_{s_{F L L}}$ ).

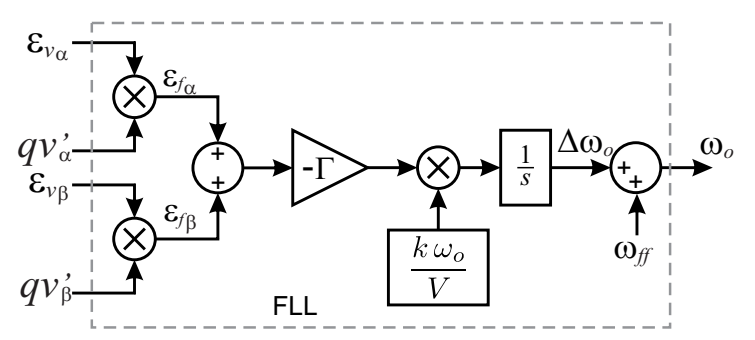

Fig. 3. FLL for frequency estimation and frequency adaptation for the SOGI. 


\section{Limit Comparators}

The output signals $V_{f}^{+}, V_{f}^{-}$and $\omega_{o_{f}}$ are compared with their respective limits. If the value of one of these variables is inside the permissible range, its respective comparator output is 0 , otherwise, it is 1. Hysteresis comparators are used in order to avoid incorrect fault detections due noise and interference. The comparators' outputs $\left(C_{V^{+}}, C_{V^{-}}\right.$and $\left.C_{w}\right)$ as functions of their inputs are shown in Figure 4. Considering the positivesequence comparator, when $V_{f}^{+}$becomes lower than $V_{M I N}$, $C_{V^{+}}$becomes 1 indicating a fault condition. After that, $C_{V^{+}}$ is still in 1, even if $V_{f}^{+}$increases above $V_{M I N}$. It will return to zero only if $V_{f}^{+} \geq V_{M I N_{h}}$. The upper limit detection behavior for $V_{f}^{+}$is similar. The negative-sequence comparator has only the upper limit detection, since its nominal value is zero. The frequency comparator has lower and upper limit detections, similar to the positive-sequence comparator.

The fault detector output, $F D$, is the logical sum of the comparators' outputs, i.e.,

$$
F D=C_{V^{+}}+C_{V^{-}}+C_{w} .
$$

Thus, $F D=0$ in grid normal condition and $F D=1$ when the electrical grid does not meet the predefined requirements.

The overall diagram of the proposed fault detection system is shown in Figure 5. The three-phase voltages at the PCC are measured and converted to the $\alpha \beta$ reference frame and one SOGI is used to obtain the in-quadrature component of each voltage component $\left(q v_{\alpha}\right.$ and $\left.q v_{\beta}\right)$. The positive- and negativesequence components are obtained by (3) and (4), respectively. After that, the magnitudes of these components are filtered by $2^{n d}$-order LPF. The FLL estimated frequency is also filtered by a $2^{\text {nd }}$-order LPF, but, in this case, with a lower cutoff frequency. Finally, each variable is applied to its respective hysteresis comparator. The outputs of these comparators are logically summed to determine the electrical grid state (normal or under failure).

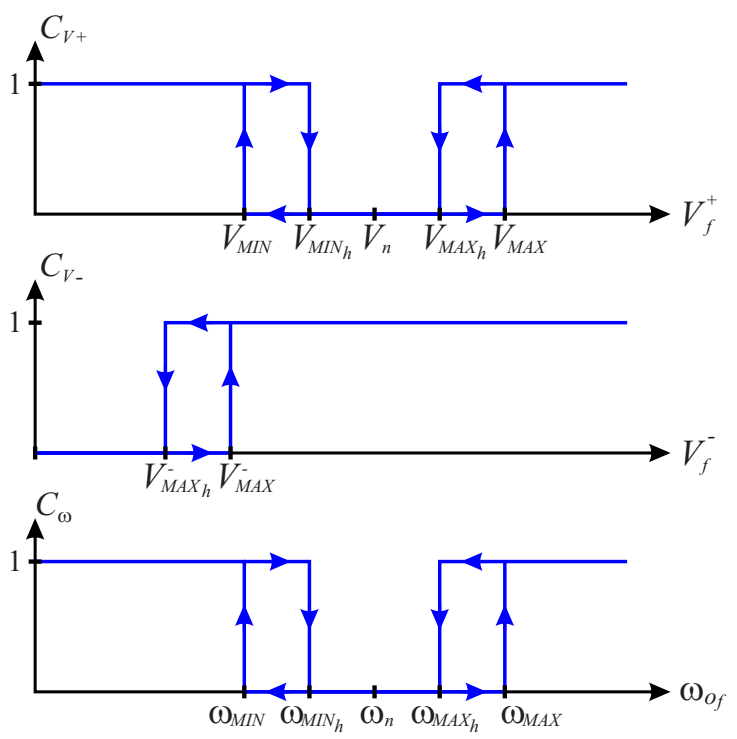

Fig. 4. Characteristics of the comparators.

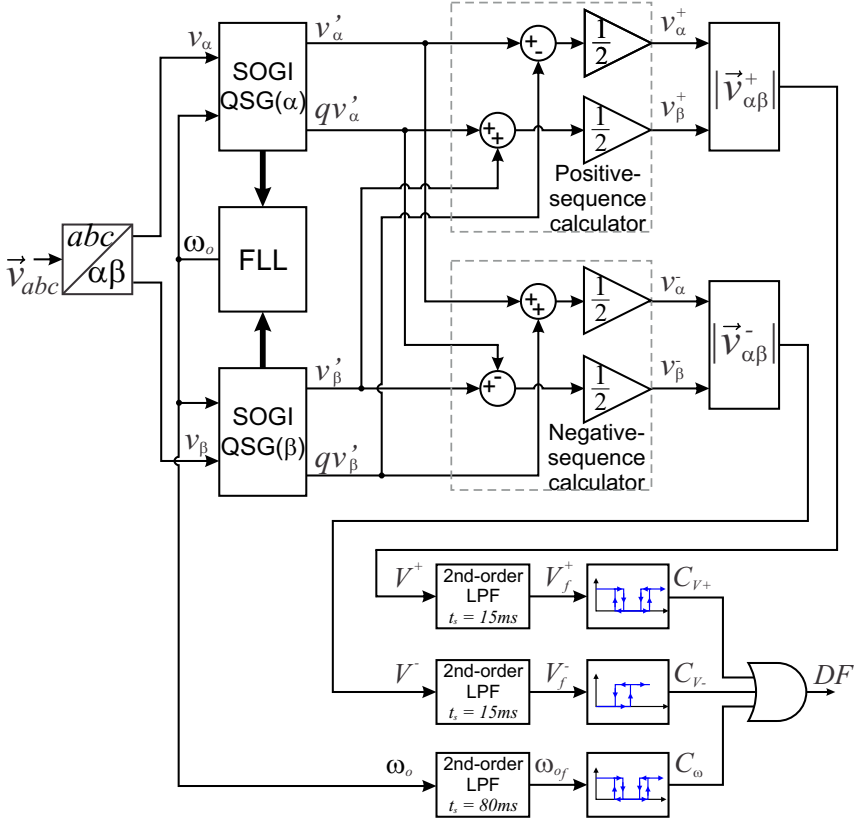

Fig. 5. Diagram of the proposed fault detection system.

\section{SOGI Discretization}

There are many methods reported in literature to discretize a continuous time-domain transfer-function. In the particular case of the SOGI, shown in Figure 1, the method consists in discretizing each one of the continuous time-domain integrators. The SOGI discretization is critical, since some discretization techniques result in behaviors that differ from the continuous time-domain response. Better results are obtained with the Euler Backward integrators and computational delay, whose structure is shown in Figure 6. This discretized structure is easily implemented. The expected behavior of this discrete SOGI-QSG depends on the sampling frequency, that should be at least 50 times higher than the SOGI resonant frequency, $f_{o}[19]$.

On the other hand, the discretization of the $2^{\text {nd }}$-order LPF is not critical. Therefore, in this work, it is used the Tustin method.

\section{SIMULATION RESULTS}

The fault detection scheme was simulated in Matlab/Simulink in order to evaluate its behavior under

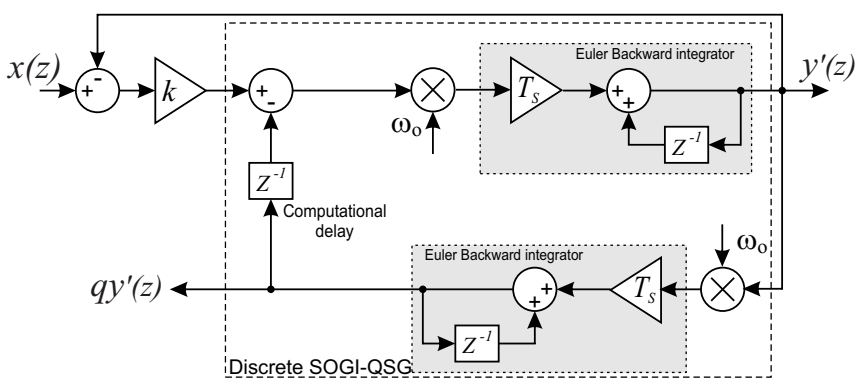

Fig. 6. Discrete SOGI-QSG based on Euler Backward integrators and computational delay. 
voltage disturbances. The main parameters used in the simulation are summarized in Table I. The fault detection algorithm was implemented in $C$ language and embedded in the Simulink using an S-Function, thus enabling its integration with the rest of the Simulink code. The grid voltages are sampled at $10 \mathrm{kHz}$ and the algorithm is executed at this same frequency. Since this frequency is much higher than the SOGI resonant frequency, the SOGI discretization, presented in Section II.D, will present the expected performance. In the first simulation, four distinct conditions are considered: initially, the voltages are balanced with nominal amplitude. After three cycles, the positivesequence voltage component drops $20 \%$ and it is restored back to its nominal value at $t=120 \mathrm{~ms}$. In the last interval of the test $(180 \mathrm{~ms} \leq t<200 \mathrm{~ms})$, the voltages become unbalanced with $20 \%$ of negative-sequence. Figure 7 shows the grid voltages in $\alpha \beta$ reference frame, the positive- and negative-sequence components extracted from the grid voltages, the estimated frequency and the fault detector output. The four simulation conditions are divided by vertical dashed lines. The horizontal dotted lines indicate the limits of the positive- and negative-sequence components and of the frequency. When the positive-sequence voltage is disturbed, both estimated negative-sequence component and frequency are disturbed as well. This occurs due the coupling among them, but these disturbances are small and they cannot exceed their respective limits. The same occurs with disturbances in negative-sequence component or in frequency. The last curve in Figure 7 shows the fault detector output, $F D$, which represents the electrical grid situation $(F D=1$ means a faulty condition). The proposed system has detected the fault condition in less than half fundamental cycle for both positive- and negative-sequence steps, as shown in Figure 7.

It should be noted that the time response depends on how severe is the fault, considering that the fault detection parameters are kept constant. In Figure 8, this behavior can be observed for balanced three-phase voltage sags. Figures 8(a) and 8 (b) show the time required by the fault detector system to identify voltage sags of $20 \%$ and $80 \%$, respectively. In the first case, the system takes $9.18 \mathrm{~ms}$ to detect the sag, whereas, for the deeper sag, it takes only $4.37 \mathrm{~ms}$.

TABLE I

\section{System parameters}

\begin{tabular}{cc}
\hline Parameter & Value \\
\hline Nominal voltage, $V_{n}$ & $220 \mathrm{~V}$ \\
\hline Nominal frequency, $\omega_{n}$ & $2 \pi 50 \mathrm{Rad} / \mathrm{s}$ \\
\hline SOGI gain, $k$ & $\sqrt{3}$ \\
\hline FLL gain, $\Gamma$ & 125 \\
\hline Settling time of the voltages $2^{n d}$-order LPF, $t_{s}$ & $15 \mathrm{~ms}$ \\
\hline Damping factor of the voltages $2^{\text {nd }}$-order LPF, $t_{s}$ & $\sqrt{3} / 2($ Bessel filter $)$ \\
\hline Settling time of the frequency $2^{\text {nd }}$-order LPF, $\xi$ & $80 \mathrm{~ms}$ \\
\hline Damping factor of the frequency $2^{\text {nd }}$-order LPF, $\xi$ & $\sqrt{3} / 2($ Bessel filter $)$ \\
\hline$V_{M A X}$ & $1.10 V_{n}$ \\
\hline$V_{M A X}$ & $1.05 V_{n}$ \\
\hline$V_{M I N}$ & $0.90 V_{n}$ \\
\hline$V_{M I N_{h}}$ & $0.95 V_{n}$ \\
\hline$V_{M A X}^{-}$ & $0.15 V_{n}$ \\
\hline$V_{M A X_{h}}^{-}$ & $0.10 V_{n}$ \\
\hline$\omega_{M A X}$ & $1.010 \omega_{n}$ \\
\hline$\omega_{M A X_{h}}$ & $1.005 \omega_{n}$ \\
\hline$\omega_{M I N}$ & $0.990 \omega_{n}$ \\
\hline$\omega_{M I N_{h}}$ & $0.995 \omega_{n}$ \\
\hline
\end{tabular}

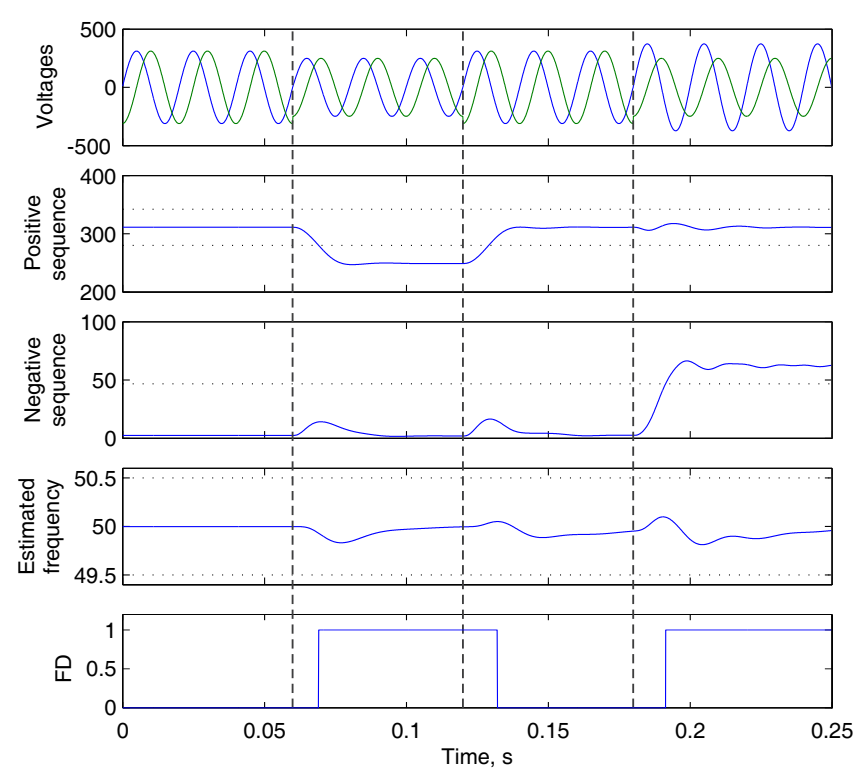

Fig. 7. Fault detection response for voltage steps in positive- and negative-sequence components.

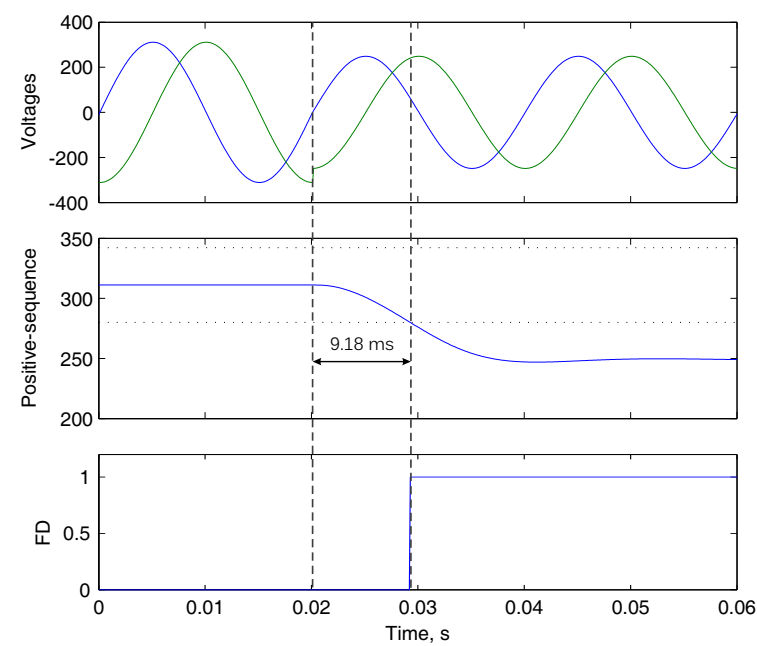

(a) Voltage sag of $20 \%$.

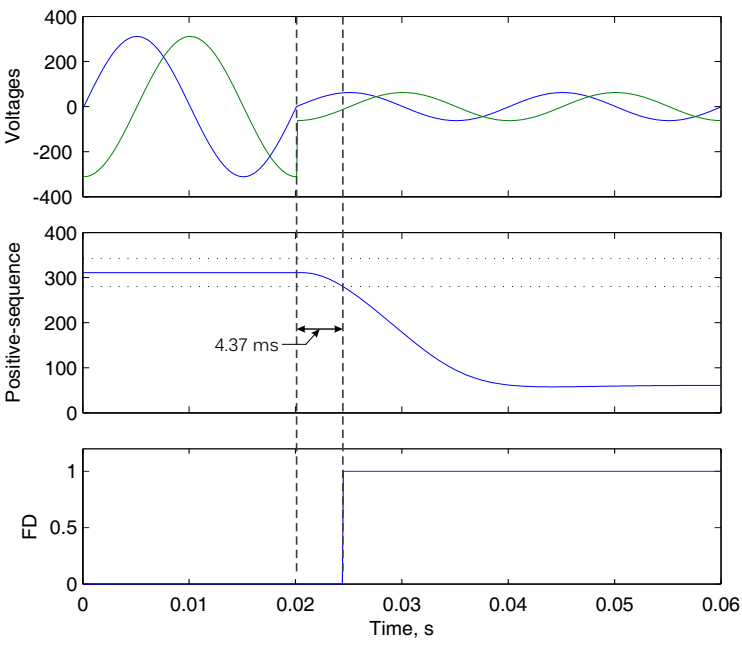

(b) Voltage sag of $80 \%$.

Fig. 8. Comparison of the fault detector actuation time as function of the voltage sag depth. 
The fault detection response for a frequency variation is shown in Figure 9. In this simulation, a step of $+1.5 \%$ in the grid frequency occurs at $t=0.6 \mathrm{~s}$. This moment is indicated by the vertical dashed line in Figure 9 . The disturbances on the voltage components due the frequency step are small. The FLL estimated frequency, $f_{o}$, and its filtered version, $f_{o f}$, are shown in Figure 9, where a zoomed view corroborates that, although the frequency detected by the FLL shows some transient oscillations, the FLL settling time matches the $40 \mathrm{~ms}$ obtained from (12). Besides, the settling time of $f_{\text {of }}$ coincides with its filter dynamics. When $f_{o f}$ exceeds its predefined limit (indicated by the horizontal dotted line), the logic variable $F D$ becomes 1 . The detection occurs in less than $40 \mathrm{~ms}$ after the frequency step, as shown in Figure 9. Therefore, it can be noted that this time response depends on how big is the frequency step.

The system's immunity to harmonics and noise was evaluated in a scenario that consists of $20 \%$ of $5^{\text {th }}$ negativesequence harmonic and $15 \%$ of $7^{\text {th }}$ positive-sequence harmonic. Besides, it is also considered a noise generated by a uniformly distributed random signal with amplitude of $10 \%$ of fundamental voltage. Thus, these distortions result in a voltage THD equal to $25.29 \%$. The triplen harmonics were not considered because they do not appear in $\alpha \beta$ reference and the high order harmonics are also not considered because they are strongly attenuated by the proposed system. This scenario is worse than that one required by the Distribution Procedures (PRODIST) of Brazilian electrical energy agency, which specify that voltage harmonics must be less than $10 \%$ in low voltage electrical grids $(<1 k V)$.

Under those circumstances, the first simulation is repeated. After three cycles, the positive-sequence voltage component drops $20 \%$ and it is restored back to its nominal value at $t=120 \mathrm{~ms}$. In the last interval $(180 \mathrm{~ms} \leq t<200 \mathrm{~ms})$, the voltages is unbalanced with $20 \%$ of negative-sequence. The grid voltages in $\alpha \beta$ reference frame are shown in Figure 10,

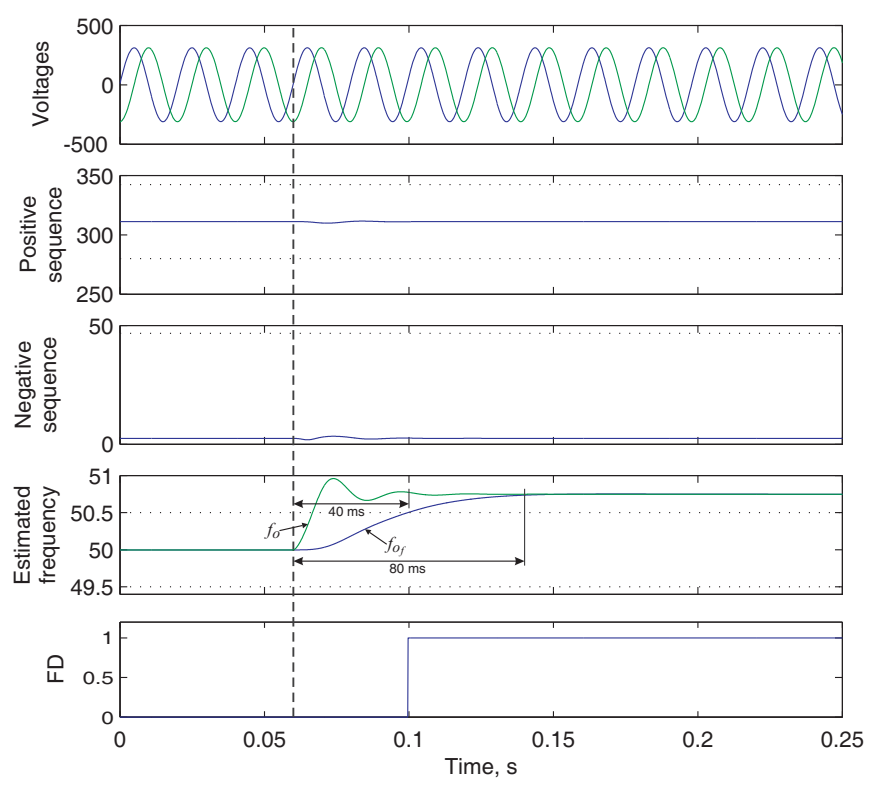

Fig. 9. Fault detection response for a $1.5 \%$ step in the grid frequency.

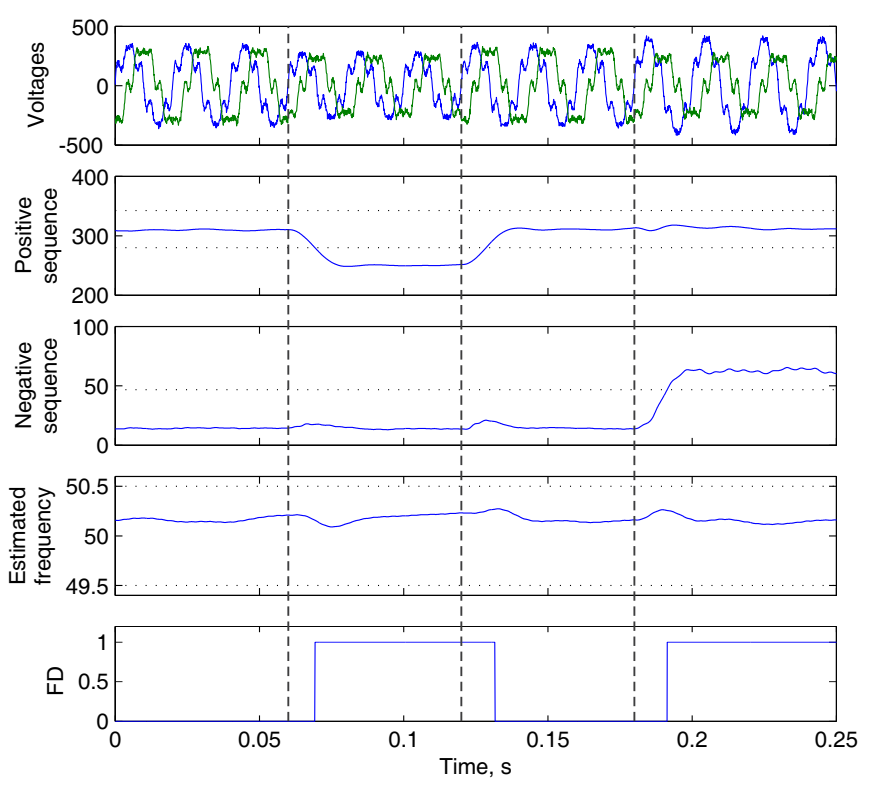

Fig. 10. Fault detection response for voltage steps in sequence components with presence of distortions and noise.

as well as, the positive- and negative-sequence components extracted from the grid voltages, the estimated frequency and the fault detector output. Note that both positive- and negativesequence components have a small distortion that does not disturb the fault detection algorithm. This high immunity to harmonics and noise is due to the SOGI, that acts as a bandpass filter for output $v^{\prime}$ and a low-pass filter for output $q v^{\prime}$, as well as the $2^{\text {nd }}$-order LPF. Although there is not voltage unbalance during the first six cycles, the DSOGI detects a negative-sequence component due to the $5^{t h}$ harmonic, which presents negative-sequence behavior. The frequency estimation is also disturbed by the harmonics and noise, but this perturbation is small and unable to cause false fault detection, as can be seen in Figure 10.

\section{EXPERIMENTAL RESULTS}

The proposed fault detection system was evaluated in an experimental set-up in which a fault is emulated by a tap change in a $Y-Y$ transformer, connected with a $\Delta-Y$ transformer, as shown in Figure 11. The fault detection algorithm is implemented in a dSpace DS1103 Control Station using the same parameters of the simulations (see Table I). The same $C$ code, used in the simulation, is also used in the dSpace real-time environment. The three-phase grid voltages

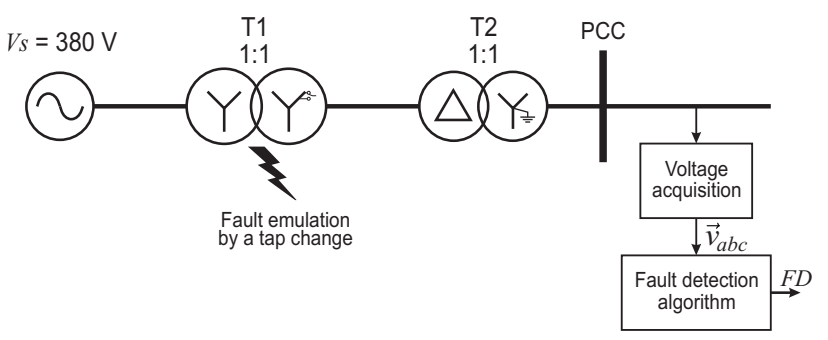

Fig. 11. Diagram of the experimental set-up. 
(phase-neutral) are sampled at $10 \mathrm{kHz}$ as in the simulation.

The fault is generated switching the tap of the grid transformer, $T 1$, in order to achieve a voltage sag of $50 \%$ on phase $a$. This single-phase fault (dip type B) is propagated to the $Y$ winding of the transformer $T 2$ as a dip type C [20]. The three-phase voltages at the PCC, during the fault, are shown in Figure 12(a). The fault is cleared after $1.2 \mathrm{~s}$. Note that the grid voltage transients are not so fast, and it takes some time to reach the steady-state operation. The positivesequence component and its magnitude, detected by the fault detector system, are shown if Figure 12(b). These results show the good performance of the DSOGI-FLL as positiveand negative-sequence voltage detector. Besides, these results are in agreement with the simulations presented before. The deviation around the estimated frequency, i.e., the output of the FLL integrator (see Figure 3), is shown in Figure 12(c). The large oscillation in $\Delta f_{o}=\Delta \omega_{o} /(2 \pi)$, when the fault is cleared, is due the slow transient dynamics of the grid voltages at the PCC. The three-phase voltages and the fault detector output, $F D$, are plotted in Figure 13, for the same fault conditions described in Figure 12. It can be verified the fast response of the proposed detection system (approximately $6 \mathrm{~ms}$ ). Figure 14 shows another experimental result, in which it is possible to note the behavior of the fault detector scheme for a complete loss of the grid voltages.

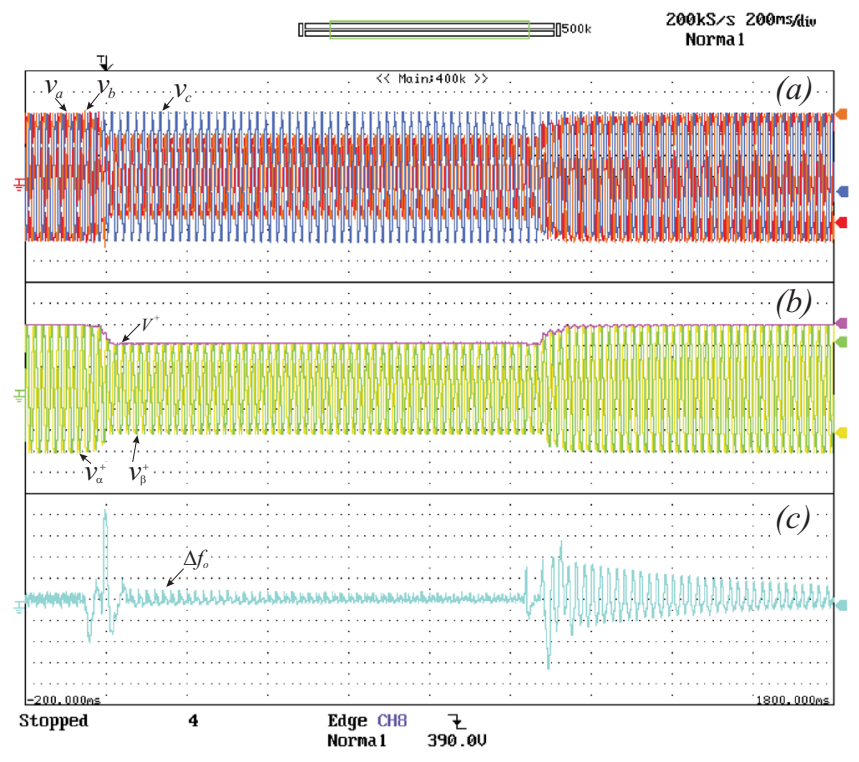

Fig. 12. Positive-sequence detection and frequency estimation with the DSOGI-FLL. (a) Three-phase voltages 100V/div; (b) Positive sequence components 100V/div; $(c)$ Estimated frequency $0.5 \mathrm{~Hz} / \mathrm{div}$.

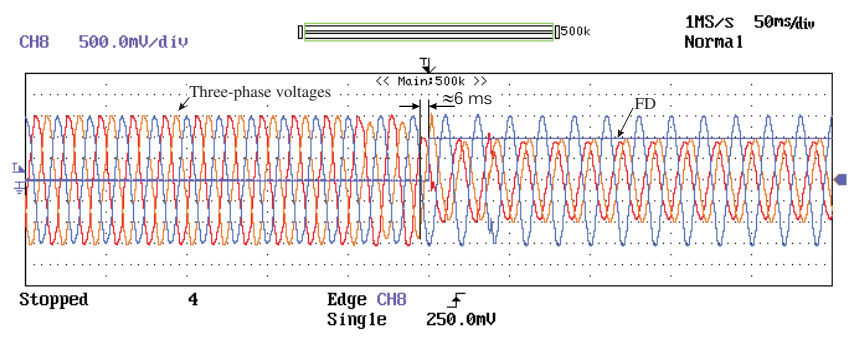

Fig. 13. Detection of a single-phase fault. Three-phase voltages $100 \mathrm{~V} /$ div; Fault detector output.

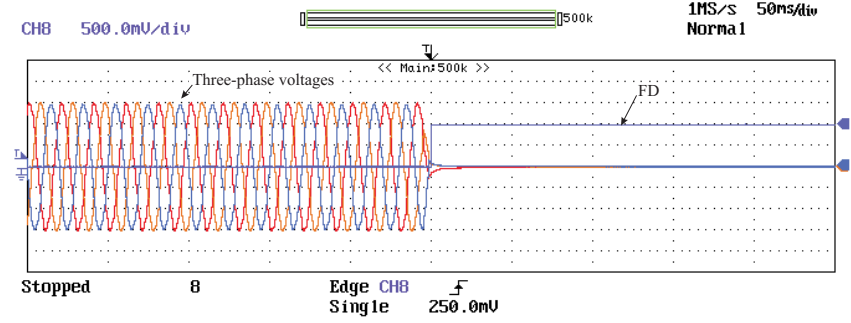

Fig. 14. Detection of a voltage loss. Three-phase voltages 100V/div; Fault detector output.

\section{CONCLUSION}

This paper proposes a fault detection algorithm based on a DSOGI-FFL, in order to obtain the voltage components as well as the frequency at the PCC. Therefore, a faulty condition is identified based on the voltage characteristics instead of using the short-circuit current amplitude. The parameters of the DSOGI-FFL should be tuned in order to achieve a fast dynamic response. Additionally, low-pass filters are used in each output signal of the DSOGI-FLL, in order to reduce the influence of noises and harmonics and set the desired dynamic responses. Finally, hysteresis comparators with their predefined limits are used to determine the grid condition: normal or faulty. The proposed system has a good time response and noise immunity. Besides, due to its simplicity, the fault detection scheme can be implemented in any digital signal processor. The simulation and experimental results validate the mathematical approach and demonstrate the effectiveness and reliability of the proposed detection system.

\section{ACKNOWLEDGEMENT}

This work was supported by the Coordenação de Aperfeiçoamento de Pessoal de Nível Superior (CAPES) and by the Conselho Nacional de Desenvolvimento Científico e Tecnológico $(\mathrm{CNPq})$, Brazil. The authors also would like to thanks the Renewable Electrical Energy Systems (REES) research group at the Technical University of Catalonia (UPC), Spain, for their support in the experiments.

\section{REFERENCES}

[1] International Energy Outlook 2010. Technical Report DOE/EIA-0484(2010), U.S. Energy Information Administration - EIA, Jul. 2010.

[2] F. Katiraei and M. R. Iravani. Power management strategies for a microgrid with multiple distributed generation units. IEEE Trans. Power Systems, 21(4):1832-1841, Nov. 2006.

[3] S. Chowdhury and S. P. Chowdhury and P. Crossley. Microgrids and Active Distribution Networks. The Institution of Engineering and Technology, London, U. K., 1st edition, 2009.

[4] M. C. Chandorkar and D. M. Divan and R. Adapa. Control of parallel connected inverters in standalone ac supply systems. IEEE Trans. Ind. Appl., 29(1):136-143, Jan./Feb. 1993. 
[5] E. A. A. Coelho and P. C. Cortizo and P. F. D. Garcia. Small-signal stability for parallel-connected inverters in stand-alone ac supply systems. IEEE Trans. Ind. Appl., 38(2):533-542, Mar./Apr. 2002.

[6] Y. A. R. I. Mohamed and E. F. El-Saadany. Adaptive decentralized droop controller to preserve power sharing stability of paralleled inverters in distributed generation microgrids. IEEE Trans. Power Electron., 23(6):28062816, Nov. 2008.

[7] E. Barklund and N. Pogaku and M. Prodanovic and C. Hernandez-Aramburo and T. C. Green. Energy management in autonomous microgrid using stabilityconstrained droop control of inverters. IEEE Trans. Power Electron., 23(5):2346-2352, Nov. 2008.

[8] K. D. Brabandere and B. Bolsens and J. V. D. Keybusand and A. Woyte and J. Driesen. A voltage and frequency droop control method for parallel inverters. IEEE Trans. Power Electron., 22(4):1107-1115, Jul. 2007.

[9] Y. Li and Y.W. Li. Decoupled power control for an inverter based low voltage microgrid in autonomous operation. In IEEE 6th Int. Power Electr. and Motion Control Conf. - IPEMC'09, pages 2490-2496, Wuhan, China, May 2009.

[10] J. M. Guerrero and J. Matas and L. G. Vicuña and M. Castilla and J. Miret. Decentralized control for parallel operation of distributed generation inverters using resistive output impedance. IEEE Trans. Ind. Electron., 54(2):994-1004, Apr. 2007.

[11] Semiconductor Equipment and Materials International. SEMI F47-0200 - Specification for Semiconductor Processing Equipment Voltage Sag Immunity, 1999/2000.

[12] A. Kusko and M. T. Thompson. Power Quality in Electrical Systems. McGraw-Hill, 1st edition, 2007.

[13] W. E. Feero and D. C. Dawson and J. Stevens. Protection Issues of the Microgrid Concept. White Paper, U.S. Department of Energy, Mar. 2002.

[14] C. L. Fortescue. Method of symmetrical co-ordinates applied to the solution of polyphase networks. Trans. of the American Institute of Electrical Engineers, (2):10271140, Jul. 1918.

[15] W. V. Lyon. Application of the Method of Symmetrical Components. McGraw-Hill, New York, USA, 1937.

[16] X. Yuan and W. Merk and H. Stemmler and J. Allmeling. Stationary-frame generalized integrators for current control of active power filters with zero steady-state error for current harmonics of concern under unbalanced and distorted operating conditions. IEEE Trans. Ind. Appl., 38(2):523-532, Mar. 2002.

[17] P. Rodriguez and A. Luna and M. Ciobotaru and R. Teodorescu and F. Blaabjerg. Advanced grid synchronization system for power converters under unbalanced and distorted operating conditions. In The 32nd Annual Conference of the IEEE Industrial Electronics Society - IECON'06, pages 5173-5178, Paris, France, Nov. 2006.

[18] P. Rodriguez and A. Luna and I. Candela and R. Teodorescu and F. Blaabjerg. Grid synchronization of power converters using multiple second order generalized integrators. In The 34th Annual Conference of the IEEE Industrial Electronics Society - IECON'08, pages 755760, Orlando, USA, Nov. 2008.

[19] F. J. Rodriguez and E. Bueno and M. Aredes and L. G. B. Rolim and F. A. S. Neves and M. C. Cavalcanti. Discrete-time implementation of second order generalized integrators for grid converters. In The 34th Annual Conference of the IEEE Industrial Electronics Society - IECON'08, pages 176-181, Orlando, USA, Nov. 2008.

[20] G. M. S. Azevedo and P. Rodriguez and M. C. Cavalcanti and G. Vazquez and F. A. S. Neves. New control strategy to allow the photovoltaic systems operation under grid faults. In The 10th Brazilian Power Electronics Conference - COBEP 2009, pages 196-201, Bonito, Brazil, Sep./Oct. 2009.

\section{BIOGRAPHIES}

Gustavo M. S. Azevedo was born in Belo Jardim, Brazil, in 1981. He received the B.Sc., M.Sc. and Ph.D. degrees in electrical engineering from the Federal University of Pernambuco in 2005, 2007 and 2011, respectively. He worked as a visiting scholar at the Polytechnical University of Catalunya, Barcelona, Spain, from 2008 to 2009. Currently, he is working as a substitute professor in the Department of Electrical Engineering at the Federal University of Pernambuco. His research interests are renewable energy systems and microgrids.

Marcelo C. Cavalcanti was born in Recife, Brazil, in 1972. He received the B.Sc. degree in electrical engineering in 1997 from the Federal University of Pernambuco, Recife, Brazil, and the M.Sc. and Ph.D. degrees in electrical engineering from the Federal University of Campina Grande, Campina Grande, Brazil, in 1999 and 2003, respectively. He worked as a visiting scholar at Center for Power Electronics Systems, Virginia Polytechnic Institute and State University, Blacksburg, USA, from October 2001 to August 2002. Since 2003, he has been at the Department of Electrical Engineering, Federal University of Pernambuco, where he is currently a Professor of Electrical Engineering. His research interests are renewable systems and power quality.

Francisco A. S. Neves was born in Campina Grande, Brazil, in 1963. He received the B.Sc. and M.Sc. degrees in electrical engineering from the Federal University of Pernambuco, Recife, Brazil, in 1984 and 1992, respectively, and the Ph.D. degree in electrical engineering from the Federal University of Minas Gerais, Belo Horizonte, Brazil, in 1999. $\mathrm{He}$ worked as a visiting scholar at Georgia Institute of Technology, USA, during 1999, and at University of Alcalá, Spain, from February 2008 to January 2009. Since 1993, he has been with the Department of Electrical Engineering, Federal University of Pernambuco, where he is currently a Professor of Electrical Engineering. His research interests include power electronics, renewable energy systems, power quality and grid synchronization methods.

Fabrício Bradaschia was born in São Paulo, Brazil, in 1983. He received the B.Sc., M.Sc. and Ph.D. degrees 
in electrical engineering from the Federal University of Pernambuco, Recife, Brazil, in 2006, 2008 and 2012, respectively. From August 2008 to August 2009, he worked as a Visiting Scholar at the University of Alcalá, Madrid, Spain. Currently, he is working as a substitute professor in the Department of Electrical Engineering at the Federal University of Pernambuco. His research interests are Z-source converters, renewable energy systems and grid synchronization methods. 